\title{
Perception of Pain during Orthodontic Treatment among the General Public Undergoing and Not Undergoing Orthodontic Treatment
}

\author{
Bhavani Ganesan¹, Jaiganesh Ramamurthy² \\ 1Department of Orthodontics, Saveetha Dental College and Hospitals, Saveetha Institute of \\ Medical and Technical Sciences (SIMATS), Saveetha University Chennai, Tamil Nadu, India. \\ 2Department of Periodontics, Saveetha Dental College and Hospitals, Saveetha Institute of Medical \\ and Technical Sciences (SIMATS), Saveetha University Chennai, Tamil Nadu, India.
}

\section{ABSTRACT}

\section{BACKGROUND}

Orthodontic treatment provides better alignment of teeth and improves occlusal and temporomandibular joint relationship. It also improves facial aesthetics, phonetics thereby improving the overall well-being of the individual. It is common to see patients to have dull aching pain and mild discomfort while undergoing orthodontic treatment. The main cause of pain during orthodontic treatment is the application of forces to activate tooth movement, placement of separators, and while debonding. The aim of the study was to evaluate the perception of pain in orthodontic treatment among school children who are undergoing and not undergoing orthodontic treatment.

\section{METHODS}

The school students were divided in to two groups. Group 1 consists of students who are undergoing orthodontic treatment and group 2 consists of students who are not undergoing orthodontic treatment. Questionnaire forms will be given to both the groups. Questionnaire forms were different in both the groups.

\section{RESULTS}

The school student's contest of two groups: group 1 consist of students who are undergoing orthodontic treatment and group 2 consist of students who are not undergoing orthodontic treatment. Questionnaire forms will be given to both the group of students.

\section{CONCLUSIONS}

This study helped in understanding the perception of pain which is felt by the school students who are undergoing orthodontic treatment and also the perception of pain related to orthodontic treatment who are not undergoing orthodontic treatment. Students who are not undergoing orthodontic treatment has a strong perception towards pain during orthodontic treatment and it is the role of the dentist to create more awareness among the public about the real facts about the orthodontic treatment to prevent the mental stigma about pain. This will help the patients especially school going children to show positive approach in undergoing orthodontic treatment.

\section{KEY WORDS}

Pain, Orthodontics, Treatment, School Students
Corresponding Author: Dr. Jaiganesh Ramamurthy, Professor, Department of Periodontics, Saveetha Dental Collage and Hospitals, 162, Poonamalee High Road, Chennai-600077, Tamil Nadu, India. E-mail: dr.r.jaiganesh@gmail.com

DOI: $10.14260 / \mathrm{jemds} / 2020 / 569$

How to Cite This Article:

Ganesan B, Ramamurthy J. Perception of pain during orthodontic treatment among the general public undergoing and not undergoing orthodontic treatment. J Evolution Med Dent Sci 2020;9(36):26142619, DOI: $10.14260 /$ jemds/2020/569

Submission 03-02-2020,

Peer Review 27-07-2020,

Acceptance 03-08-2020,

Published 07-09-2020.

Copyright (c) 2020 JEMDS. This is an open access article distributed under Creative Commons Attribution License [Attribution 4.0 International (CC BY 4.0)] 


\section{BACKGROUND}

Orthodontics is the branch of dentistry that corrects teeth and jaws that are positioned improperly. Orthodontic treatment provides better alignment of the teeth and improves the occlusal and TMJ relationship. This not only helps in better mastication, phonetics and facial aesthetics, but also contributes to general well-being and oral health, thereby improving the quality of life. Orthodontics is concerned with the development of the dentition, facial growth and occlusion together with the prevention and correction of occlusal anomalies. Diagnosis and treatment planning are of utmost importance for providing a desired result for the patient. Softtissue examination has become a crucial segment of orthodontic diagnosis and treatment planning. Like any other treatment procedure, orthodontic treatment, in addition to its therapeutic advantages it is also associated with risks and complications. However, when you weigh the risk and complication associated with treatment to other alternative, orthodontic treatment have considerably lower complications compared to other surgical or nonsurgical intervention. ${ }^{1}$ Orthodontic treatment in adults has gained social and professional acceptance in recent years. The intention to improve dentofacial aesthetics was the main motivation commonly expressed by orthodontic patients. The fact that malocclusion is not a disease and a patient's decision to undergo orthodontic treatment is purely subjective and influenced by patient and care provider factors. An assessment of orthodontic treatment need helps to identify individuals who will benefit from treatment and safeguard their interest. It is common to see patients to have dull aching Pain and mild discomfort while undergoing orthodontic treatment. Patients who underwent both premolar extractions and orthodontic tooth movement experienced more pain 24 hours after initial archwire placement than 24 hours after tooth extraction. Complicating this issue is the evidence of individual consistency in both pain threshold and pain response. ${ }^{1}$

Malocclusion is defined as the absence of an association between the upper and lower arches and abnormal alignment of the teeth. It has led to an increased interest in orthodontic treatment in many countries. Malocclusion can increase the risk of dental trauma, caries, periodontal problems, and oral dysfunctions such as chewing, swallowing, and talking difficulties. While malocclusion has been identified not as disease but morphological variation, others have referred to it not as a disease problem by some. Class II malocclusion which defines as maxilla and mandible skeletal disharmony with underdevelopment of mandibular growth. The clinical features of Class II malocclusion are increased overjet, anterior crowding and upper molar distalization. This can lead to several periodontal problems such as early tooth loss and periodontal pockets. ${ }^{2}$ Malocclusion not only affects the appearance of an individual but also influences in many other ways such as interference with normal growth and development, improper or abnormal muscle function, speech defects, increased carried incidence, prediction to periodontal disease, and temporomandibular joint disorder. One of the most common types of malocclusions that affect the aesthetic appearance, function, and quality of life of the patients is dental crowding. Malaligned teeth often result into food lodgement aesthetic and psychological problems in patients.
Dental arch dimensions including dental arch width, arch length and arch form play an important role in diagnosis, treatment planning and outcomes in orthodontic treatments. There are several factors that may be affected by dental arch forms such as available space, smile aesthetics and potentially long-term occlusal stability. During orthodontic treatment, maintaining the pre - treatment dental arch form may help to reduce crowding relapse and periodontal damage. Dental arch forms may be varied based on ethnic groups and populations. ${ }^{3}$

The most commonly reported adverse effects of orthodontic treatment can be both local and systemic. This includes, pain, tooth discolorations, decalcification, root resorption, periodontal complications, psychological disturbances, gastrointestinal complications, allergic reactions, infective endocarditis, and chronic fatigue syndrome. The main cause of pain during orthodontic treatment is the application of forces to activate tooth movement, placement of tooth separators, and while debonding. 4 Pain is influenced by gender, behavioural factors, individual pain threshold, magnitude of force that is delivered, and motivation. . $^{3-5} \mathrm{TMJ}$, soft tissues like lips, gingiva and tongue are not affected by fixed appliances. ${ }^{6-8}$ Discomfort is mainly localized at the individual or set of teeth. Among the soft tissues, Ulcer sites are localized at the side of the buccal mucosa.9,10 It has been observed that well - informed patients has lesser pain perception and require lesser number of medications. The aim of this present study was to evaluate perception of pain towards orthodontic treatment by questionnaire survey among the school students who are undergoing orthodontic treatment and those not undergoing orthodontic treatment.

\section{METHODS}

This questionnaire based descriptive study was conducted among 100 patients who were divided into two groups. Group I consist of 50 people who are undergoing orthodontic treatment and Group II consist of 50 people who are not undergoing orthodontic treatment. This is done in a school where students from the same level who are undergoing orthodontic treatment and who are not undergoing orthodontic treatment to minimize the margin of error. The questionnaire form was relevant to pain perception toward orthodontic treatment. For evaluation of perception pain, there were 2 questionnaires one for each group because the questions vary in patients undergoing orthodontic treatment group and not undergoing orthodontic treatment. Every individual develops mental perception about the things they observe. Patients develop a perception of pain when they see others who are undergoing treatment. Orthodontic treatment involves wires, brackets and elastics which gives a sense of pain among those who are not undergoing treatment. Questionnaire forms were given for validation to another orthodontist who is not part of the study.

\section{Statistical Analysis}

The questionnaire data was collected and analysed statistically. Number of positive responses to negative response to total number of participants were calculated as 
percentage and graphically represented in the form of bar charts below. Group I data was analysed in graph 1, 2 and 3 and Group II data was analysed in graph 4, and 5.

\section{RESULTS}

\section{Group 1}

In group 1, all the patients who were undergoing orthodontic treatment had pain during orthodontic treatment and few patients took pain killer medications for pain relief and diet pattern was affected in significant numbers. Its represented graphically in Graph I. Graph I Presents $100 \%$ of the people reported that there was pain during the orthodontic treatment. $10 \%$ of the people have consumed pain reliever during orthodontic treatment and $90 \%$ of the people have not consumed pain reliever during treatment. $60 \%$ of the people has a disturbance in their diet and $40 \%$ of the people has no disturbance in their diet.

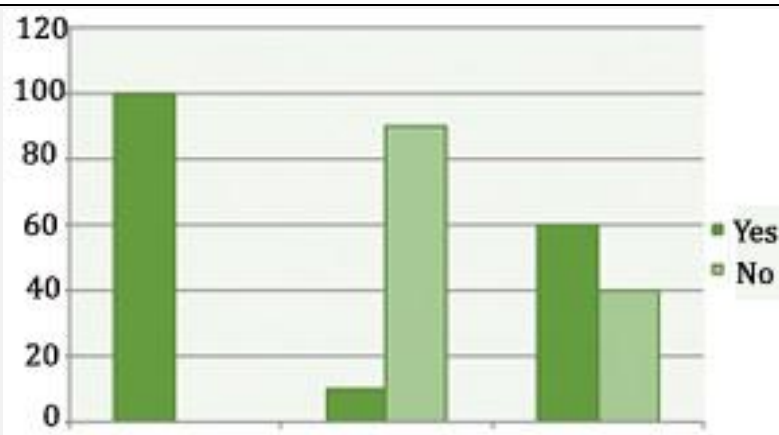

Do you have pain Do you consumed Is your diet as during orthodontic any pain reliever? been affected? treatment?

Graph 1. Percentage of people who had pain during orthodontic treatment and percentage of people who consumed pain reliever drug after treatment and people affected with their diet pattern

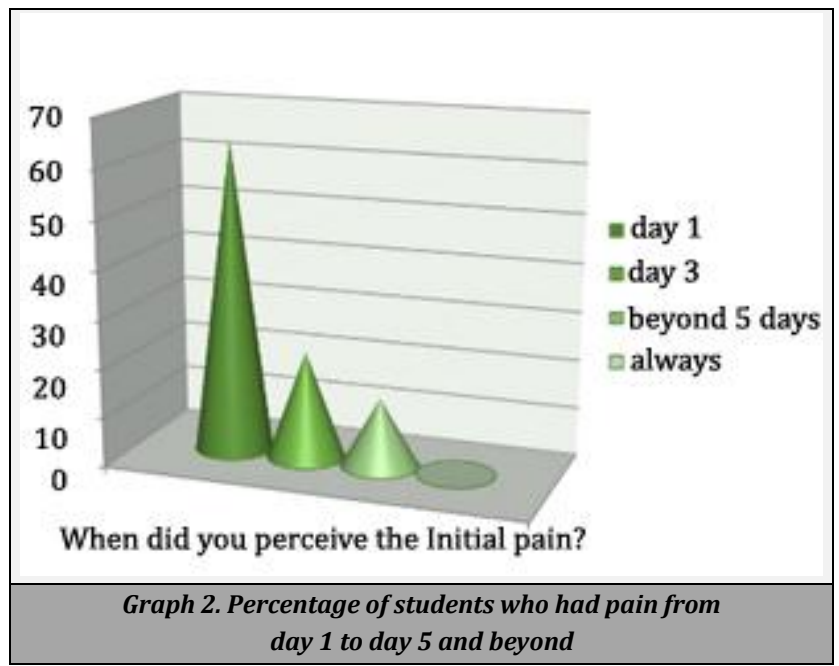

Patients who had pain during orthodontic treatment vary with number of days they suffered. Significant number of participants developed pain in first day and reduced gradually. Graph 2: Represents $64 \%$ of the people felt that they perceived the initial pain at day 1, $22 \%$ of the people felt that they had pain till day 3 and $14 \%$ of the people felt that they perceived the initial pain at beyond day 5 and no one felt that they had pain always.

Participants who had pain differed in the region of pain. Around $10 \%$ of the people feel that they had a pain in front teeth region, $25 \%$ of the people feel that they had a pain in back teeth region, $65 \%$ of the people that they had experienced a pain in both front and back teeth region. It is represented graphically in Graph 3.

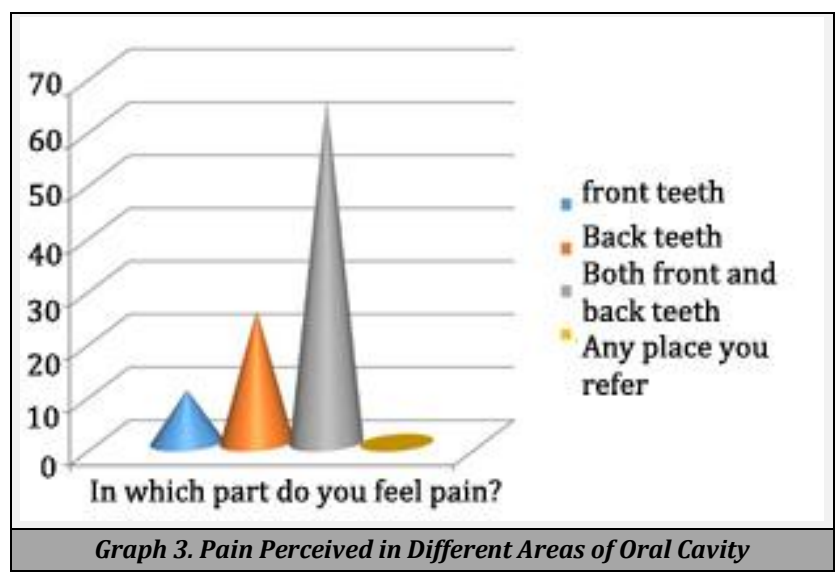

\section{Group 2}

In group 2, patients didn't undergo orthodontic treatment and questions were asked about the awareness of malocclusion, ways of correcting malocclusion, awareness about orthodontic treatment. Around $76 \%$ of the people reported that they were aware of their teeth is not aligned properly and $24 \%$ of the people reported that they were not aware about their teeth is not aligned properly. $54 \%$ of the people reported that they were aware of their teeth can be corrected and $46 \%$ reported that they were not aware of their teeth can be corrected. $64 \%$ of the people reported that they were aware about the orthodontic treatment available and $36 \%$ of the people reported that they were not aware about the orthodontic treatment available.

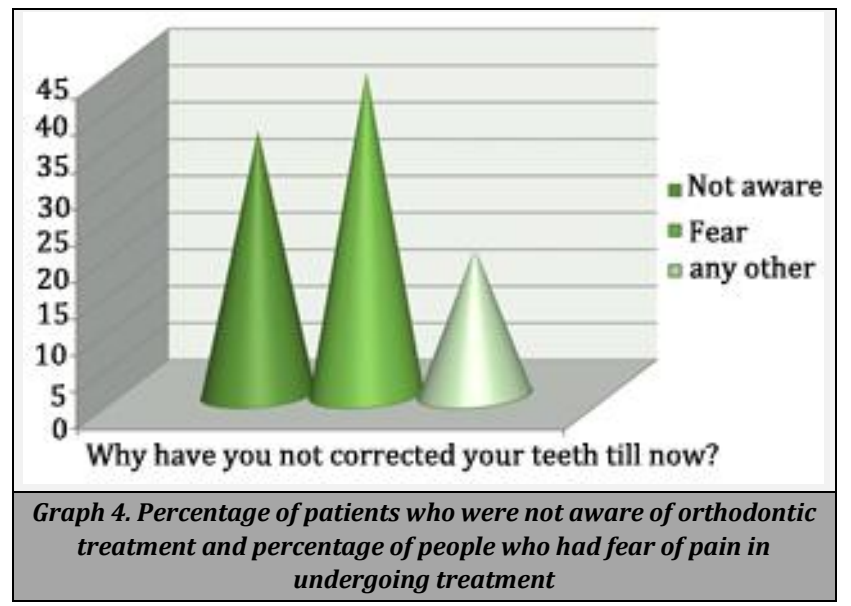

When they were asked why orthodontic treatment was not opted even after knowing the teeth malalignment, $36 \%$ of the people reported that they were not aware of the treatment for correcting their teeth, $22 \%$ of the people reported that they had fear of the treatment for correcting their teeth and $10 \%$ 
of the people had some other reason of not correcting their teeth. Its graphically represented in Graph 4.

When analysed for perception of pain towards orthodontic treatment, $68 \%$ of the people felt orthodontic treatment is painful and $32 \%$ of the people felt that orthodontic treatment is not painful and represented graphically in Graph 5.

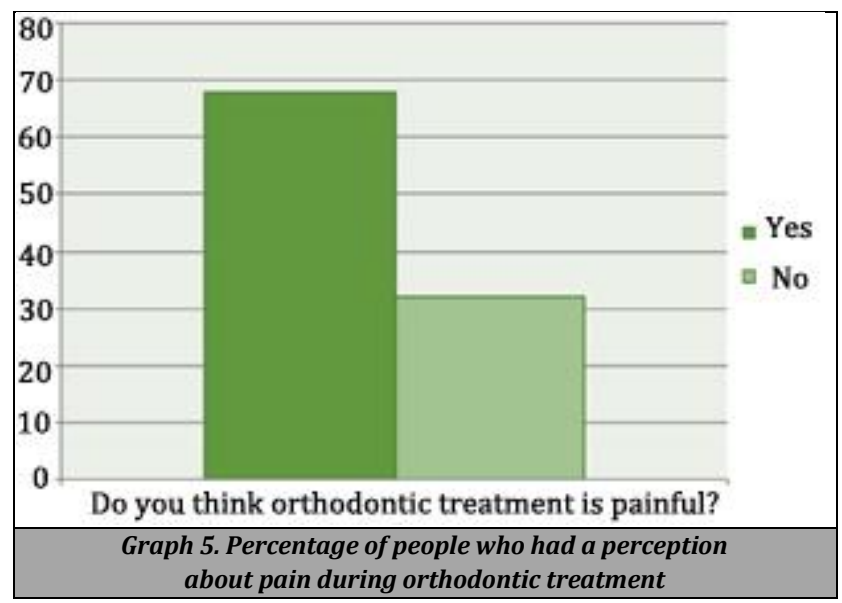

\section{DISCUSSION}

Malocclusion is the misalignment or incorrect relation between the teeth and the dental arches. It is one of the leading causes of progression towards poor oral hygiene. Fixed orthodontic treatment is the well accepted and most reliable method for treating malocclusion. Fixed appliances use orthodontic brackets, orthodontic bands for fixed retention which can complicate patient's routine oral hygiene, and this can cause accumulation of dental biofilm and gingival inflammation. Aesthetics is the major concern in patients who opt for orthodontic treatment and it has been found that gummy smile is commonly detected aesthetic problem affecting younger age groups due to variety of causes such as skeletal, dento-alveolar, or soft-tissue alterations. It can be due to altered development of jaw, altered passive eruption, or malpositioning of teeth. Orthodontic tooth movement is dependent on efficient remodelling of bone. The cell - cell interactions are understood in molecular level in recent years and the links between osteoblasts and osteoclasts appear to be controlled by the production and responses of osteoprotegerin ligands. ${ }^{11}$ The theories of orthodontic tooth movement remains debatable, but the histological documentation is beyond any doubts. A periodontal ligament subjected to pressure will result in bone resorption whereas a periodontal ligament under tension results in bone formation. Frequent occlusal trauma increases the burden to transmission of occlusal force and often leads to resorption of alveolar bone. When excessive, abnormal occlusal force is removed and normal bite force is restored through orthodontic treatment, alveolar bone is rehabilitated with increased bone density. However, in addition to the increased occlusal force abnormal occlusal force also comes under reduced occlusal function. Orthodontic treatment is associated with a number of adverse effects, such as root resorption, pain, pulpal changes, periodontal disease, and temporomandibular dysfunction (TMD). This could be a result of reduced oral hygiene in molar regions and the presence of molar bands, which can harbour food leading to food lodgement. ${ }^{12}$

Orthodontic treatment is known to affect the balance of oral microbial flora by increasing bacterial adhesion and retention. ${ }^{11-13}$ The effect of orthodontic treatment on the periodontal connective tissues for longer duration is questionable. Alveolar bone loss may be detected during or immediately after completion of orthodontic treatment. It is well accepted fact that reduced oral hygiene measures have resulted in enamel decalcification and gingival inflammation. Such gingival inflammation has a negative impact for the young adult who is undergoing orthodontic treatment and should be minimized if possible. The niches for bacterial accumulation can be eradicated by using the one-stage, fullmouth disinfection protocol before initiating orthodontic treatment. It has been observed from studies that an increase in the value of periodontal indices and growth of periodontal pathogenic bacteria were observed in adolescent patients undergoing fixed orthodontic treatment. In the majority of the patients, following placement of a fixed appliance, small amounts of gingival inflammation is visible, which could be for shorter duration in nature and does not lead to clinical attachment loss. Oral cavity comprises more than 700 different species of microorganisms of which significant numbers exist as commensals. Some exist as opportunistic organisms. Streptococcus mutans is a commensal of the microbiota of the oral cavity, has been detected in $70 \%$ of the healthy subjects and in almost $90 \%$ of a periodontal diseases group along with different species like Porphyromonas gingivalis specifically linked to the cause of periodontitis by a specific PCR assay.

Streptococcus mutans, Staphylococcus species are also the most common bacteria found in the supra gingival region of the patients undergoing orthodontic treatment and along with pathogenic organisms like P. gingivalis, which on a long-term effect can cause destruction of the supporting structures of the periodontium. Gingivitis is the inflammation of the gingival tissue which occurs in 2-3 days after the plaque formation and microorganisms present in the plaque triggers host inflammatory response and the most predominant cell found in the gingival inflammation is the neutrophils. Before the orthodontic therapy is initiated, doctor should carefully consider the bucco - lingual thickness of the soft tissue on the pressure side of the tooth and is expected to have sufficient thickness. Furthermore, proper oral hygiene instructions for adequate plaque control measures should be given and inflammation is controlled before, during as well as after the completion of the orthodontic therapy to avoid unnecessary inflammation to the marginal gingiva. ${ }^{14}$

The manifestation of periodontal diseases results from the interaction of host defense mechanisms, microbial agents, environmental and genetic factors. Optimal periodontal health is paramount for the success of any type of orthodontic treatment, in the same way that orthodontic correction should not cause harm to periodontal connective tissues. Some of the studies in the literature investigated the interrelation between tooth malpositioning and altered occlusion when compared with periodontal health in groups of adult patients. Many studies gave importance on assessing the oral health of children and adolescents. One study examined patients 
referred to periodontal treatment by general dentist and evaluated the malpositioned teeth and their potential outcomes on periodontal tissues. Study results showed that there were abnormal periodontal changes associated with orthodontic forces, which is in accordance with the previous findings. Although the main causative factor for periodontal disease is the bacterial biofilm, other factors are also equally associated, and it leads to changes in host response of the patient. Of these accounted factors, teeth malpositioning are considered as predisposing factors because oral hygiene becomes tougher for the patient. Bacterial plaque is retained in the interdental areas and accumulates and if left untreated it's proliferates and leads to pathological periodontal changes. Various Study results has confirmed the above findings and confirmed that patients having malpositioned teeth also had some type of periodontal disease in several degrees of severity. ${ }^{15}$

The study was performed on 100 people from which 50 people who have undergoing orthodontic treatment and another 50 people who have not undergoing orthodontic treatment. From the results it has been found that $64 \%$ of the people had experienced a pain during orthodontic treatment most probably at day 1, 22 \% of the people had experienced a pain at day 3 and $14 \%$ of the people had experienced a pain at beyond 5 days. ${ }^{16}$ The people felt the pain has reduced as progress the weeks, no one experienced a pain always till orthodontic treatment completed. Periodontal pain is caused by a $10 \%$ of the people feel that they had a pain in front teeth region, $25 \%$ of the people felt that they had a pain in back teeth region, $65 \%$ of the people that they had a pain in both front and back teeth region. Now the reason of this could be that individual's threshold of pain varies from person to person. The pain seems to more initially because of the inflammatory reaction and movement of the tooth and also the pressure was felt maximum on the first day.

The forces applied could start coming down after the day 1 because of fatigue of elastic materials which are used and as the tooth moves the pressure on the tooth also reduce. The results have been found from the people who didn't undergo orthodontic treatment, of that $68 \%$ of the people felt orthodontic treatment was painful and $32 \%$ of the people felt that orthodontic treatment was not painful. $68 \%$ of the people reported that orthodontic treatment was painful from that $6 \%$ of the people reported the intensity of the pain was severe, 46 $\%$ of the people reported the intensity of the pain is moderate, $16 \%$ of the people reported the intensity of the pain is mild. Variation in pain perception could be due to individual perception to pain and the threshold to pain varies between individuals.

All the treatment in the dental specialty has its own set of complications and orthodontic therapy being no exception. Orthodontic treatment involves the application of forces that are continuous in activity on required or desired areas for the movement of dentition to the desired place with maximum comfort to the patient. ${ }^{16}$ Orthodontic treatment has no uncertainty in providing a noteworthy impact on facial aesthetics. Angle perceived that this impact could be for better or in negative ways, stating that the orthodontists "for each of his efforts, whether he realizes it or not, makes for beauty or ugliness, for harmony or disharmony, or for perfection or deformity of the face". ${ }^{17}$ Dental aesthetics are a key factor in overall physical wellbeing of the patient, which also contributes to self - esteem and personality development. This is one of the main reasons for patients to undergo orthodontic treatment for correction of malocclusion. Although orthodontic treatment has tangible advantages and benefits, including improvement in dental health, function, appearance, and self-esteem, nevertheless orthodontic appliances can cause unwanted complications also if adequate oral hygiene is not taken care off during the treatment. It is important that the patients who are undergoing orthodontic treatment should be aware of these potential risks, so that they can know their responsibilities and the expectations placed on them during the treatment. This helps in achieving successful results without any unwanted side effects during and after completion of orthodontic treatment. ${ }^{18,19}$

\section{CONCLUSIONS}

Students who are not undergoing orthodontic treatment have a strong perception towards pain during orthodontic treatment and it is the role of the dentist to create more awareness among the public about the real facts about the orthodontic treatment to prevent the mental stigma about pain. This will help the patients especially school going children to show positive approach in undergoing orthodontic treatment.

\section{Ethical Clearance}

Ethical clearance was obtained from Institutional Ethical Committee of Saveetha Dental College and Hospitals, Chennai. (IHEC / 2018 / UG - 213)

Financial or Other Competing Interests: None.

\section{REFERENCES}

[1] Jones ML. An investigation into the initial discomfort caused by placement of an archwire. Eur J Orthod 1984;6(1):48-54.

[2] Feinmann C, Ong M, Harvey W, et al. Psychological factors influencing post-operative pain and analgesic consumption. Br J Oral Maxillofac Surg 1987;25(4):28592.

[3] Kvam E, Gjerdet NR, Bondevik O. Traumatic ulcers and pain during orthodontic treatment. Community Dent Oral Epidemiol 1987;15(2):104-7.

[4] Kvam E, Bondevik, Gjerdet NR. Traumatic ulcers and pain in adults during orthodontic treatment. Community Dent Oral Epidemiol 1989;17(3):154-7.

[5] Oliver R, Knapman YM. Attitudes to orthodontic treatment. Br J Orthod 1985;12(4):179-88.

[6] Brown D, Moerenhout RG. The pain experience and psychological adjustment to orthodontic treatment of preadolescents, adolescents, and adults. Am J Orthod Dentofacial Orthop 1991;100(4):349-56.

[7] Soltis JE, Nakfoor PR, Bowman DC. Changes in ability of patients to differentiate intensity of forces applied to maxillary central incisors during orthodontic treatment. J Dent Res 1971;50(3):590-6. 
[8] White LW. Pain and cooperation in orthodontic treatment. J Clin Orthod 1984;18(8):572-5.

[9] Wilson S, Ngan P, Kess B. Time course of the discomfort in young patients undergoing orthodontic treatment. Pediatr Dent 1098;11(2):107-10.

[10] Huskisson E. Measurement of pain. Lancet 1974;2(7889):1127-31.

[11] Jones M, Chan C. The pain and discomfort experienced during orthodontic treatment: a randomised controlled clinical trial of two initial aligning arch wires. Am J Orthod Dentofacial Orthop 1992;102(4):373-81.

[12] Priyanka S, Dinesh S, Ramamurthy J. Ortho-perio relationship: is it a nuptial knot. Int J Pharma Bio Sci 2014;5(2):B100-5.

[13] Priyanka M, Jaiganesh. Periodontally accelerated osteogenic orthodontics. Int J Pharm Sci 2013;5(4):49-51.

[14] Ristic M, Svabic MV, Sasic M, et al. Clinical and microbiological effects of fixed orthodontic appliances on periodontal tissues in adolescents. Orthod Craniofac Res 2007;10(4):187-95.
[15] Ramanathan S, Ramasamy N. Avoidable complication and patient care during orthodontic treatment. J Pharma Sci Res 2015;7(12):1096-8.

[16] Dinesh SPS, Arun AV, Sundari KKS, et al. An indigenously designed apparatus for measuring orthodontic force. J Clin Diagn Res 2013;7(11):2623-6.

[17] Battagel JM. Profile changes in Class II, division 1 malocclusions: a comparison of the effects of Edgewise and Frankel appliance therapy. European J Orthodontics 1989;11(3):243-53.

[18] Kumar SB. Pain and discomfort in patients undergoing orthodontic treatment with crowding. Int J Sci Res 2017;6(10):148-50.

[19] Priyanka M, Ramamurthy J. Tissue engineering in periodontal regeneration. Res J Pharm Biol Chem Sci 2015;6(1):78-82. 